\title{
SINGULARITIES OF IMPLICIT DIFFERENTIAL SYSTEMS AND MAXIMUM PRINCIPLE
}

\author{
STANISEAW JANECZKO \\ Institute of Mathematics, Polish Academy of Sciences \\ Sniadeckich 8, P.O.B. 21, 00-956 Warszawa, Poland \\ and \\ Faculty of Mathematics and Information Science, Warsaw University of Technology \\ Pl. Politechniki 1, 00-661 Warszawa, Poland \\ E-mail: janeczko@ise.pw.edu.pl \\ FERNAND PELLETIER \\ Université de Savoie, LAMA Campus Scientifique \\ 73376 Le Bourget du Lac, France \\ E-mail: pelletier@univ-savoie.fr
}

\begin{abstract}
The integrability condition for the Lagrangian implicit differential systems of $(T P, \dot{\omega})$, introduced in [7], is applied for the specialized control theory systems. The Pontryagin maximum principle was reformulated in the framework of implicit differential systems and the corresponding necessary and sufficient conditions were proved. The beginning of the classification list of normal forms for Lagrangian implicit differential systems according to the symplectic equivalence is provided and the corresponding differential caustics are calculated.
\end{abstract}

1. Introduction. In [18] F. Takens has formulated the problem of integrability of the implicit first order differential equations. Previously such systems were introduced in physics of generalized Hamiltonian systems and statics of the constrained mechanical systems (cf. [4], [8], [5], [3]). If $P$ is a smooth manifold and $A$ is a subset of $P$, then the local integrability of a vector field $V$ on $A$ (not necessary continuous) is traditionally defined as an existence at each point $p \in A$ a $C^{1}$-curve $\gamma_{\epsilon, p}:(-\epsilon, \epsilon) \longrightarrow A$ for some $\epsilon>0$, such that $\gamma_{\epsilon, p}(0)=p$ and $\dot{\gamma}_{\epsilon, p}(t)=V\left(\gamma_{\epsilon, p}(t)\right)$ for all $t \in(-\epsilon, \epsilon)$. Such a curve is called an integral curve of $V$ with initial value $p$. Moreover we may have an extra demand that two integral curves with the same initial value are the same on their common domain, and for each $p \in A$ there is a neighbourhood $U$ of $p$ in $A$ and an $\epsilon>0$ such that the mapping

$$
U \times(-\epsilon, \epsilon) \ni(\tilde{p}, t) \mapsto \gamma_{\epsilon, \tilde{p}}(t) \in A
$$

2000 Mathematics Subject Classification: Primary 57R45; Secondary 58C35, 70H05, 34A26.

The paper is in final form and no version of it will be published elsewhere. 
is defined and continuous.

There are well known examples of discontinuous vector fields which are locally integrable in the previously defined sense (cf. [6]). The discontinuous vector fields may be constructed as subsystems of an implicit branching systems considered as the subsets (submanifolds) of the tangent bundle. Thus there is an important question for the local integrability of such systems under possible weaker assumptions.

If $f: X \longrightarrow P$ is a smooth mapping, then the class $\Xi(f)$ of natural examples of implicit parametrized differential systems is given by vector fields along the mapping $f$. $\Xi(f)$ is the set of mappings $V: X \longrightarrow T P$ such that $f=\tau_{P} \circ V$, where $\tau_{P}: T P \longrightarrow P$ is a canonical projection. If $f$ is singular then vector fields along $f$ form the implicit differential systems. For an elementary example we can take

$$
f: X \longrightarrow P, \quad f(x)=-(x-a)^{2}, \quad a \neq 0,
$$

with the singular value at $y=0$. Consider the vector field $V=x \frac{\partial}{\partial y}$ along $f$, then an implicit differential system in TP may be written in the form

$$
\dot{y}=x, \quad 0=y+(x-a)^{2}, \quad(\dot{y}, y) \in T P .
$$

If $a \neq 0$, then $V$ is not integrable at $y=0 . f$ is a locally stable mapping, then $V$ can be written in the form $V=d f \circ \xi+\eta \circ f$, where $\xi, \eta$ are the smooth vector fields (germs) in the following commuting diagram

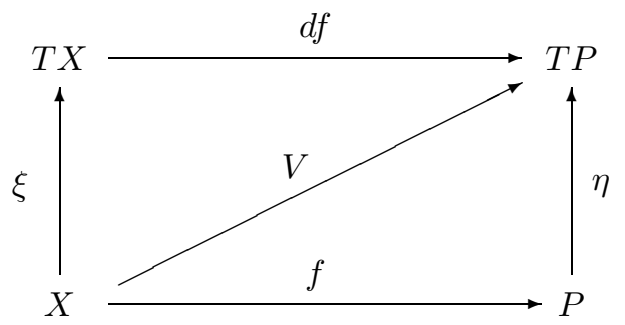

$V$ is integrable if the vector field $\eta$ is tangent to the set of critical values of $f$, but this implies the condition $a=0$.

Our main purpose in writing this paper was to show necessary and sufficient conditions for local integrability of differential systems which form the Lagrangian submanifolds in tangent bundles to symplectic manifolds. The special emphasis was done for systems which correspond to control theory systems, i.e. vector fields along sub-immersions. The complete analysis was done for the differential systems being the constrained Lagrangian submanifolds, which in the conormal bundle case correspond to the Pontryagin maximum principle (cf. [17]). The classification problem for germs of differential systems was formulated and the first normal forms of integrable Lagrangian differential systems were calculated.

Acknowledgements. We would like to thank Takuo Fukuda for helpful discussions and Jan Krzysztof Kowalski for his help in final redaction of the paper.

The first author supported by KBN grant No. 2 P03A 02017 and the special programme Dynamics of Complex Systems of Warsaw University of Technology. 
2. Implicit differential systems. Let $P$ be a smooth manifold. A submanifold $D$ of the tangent bundle $T P$ can be considered as a differential equation. If $D$ is not transversal to the fibration, then $D$ is called an implicit differential equation. We assume $\operatorname{dim} D=$ $n=\operatorname{dim} P$. This type of differential equation can be written locally in the form

$$
\begin{aligned}
\dot{x}_{i}=g_{i}(x, \lambda), & 1 \leq i \leq n, \\
0=f_{j}(x, \lambda), & 1 \leq j \leq m,
\end{aligned}
$$

where $f_{j}, g_{i}$ are smooth maps from $\mathbb{R}^{n} \times \mathbb{R}^{m}$ to $\mathbb{R}$. The most important problem, which we consider in what follows, is an existence of the integral curves of this system (cf. [18], [7], [5], [19]). More precisely:

Definition 2.1. A submanifold $D$ of $T P$ is called a first order differential system. An absolutely continuous curve $\gamma: I \longrightarrow P$ is called an integral curve of $D$ if the tangent vector $(\gamma(t), \dot{\gamma}(t))$ belongs to $D$ for almost every $t \in I$.

A differential system $D \subset T P$ is said to be integrable at $u \in D$ if there exists an integral curve $\gamma:(-\alpha, \alpha) \longrightarrow P(\alpha>0)$ of $D$ such that $(\gamma(0), \dot{\gamma}(0))=u$.

A differential system $D \subset T P$ is said to be integrable (resp. weakly integrable) if there exists a smooth (resp. absolutely continuous) integral curve at each $u \in D$.

Now we assume that $P$ is a symplectic manifold endowed with a symplectic form $\omega$. Thus we have the flat isomorphism $\beta: T P \longrightarrow T^{*} P$ of vector bundles defined by $\beta$ : $T P \ni u \mapsto u^{b}=i_{u} \omega \in T^{*} P$. Now we endow the tangent bundle TP with the symplectic structure $\dot{\omega}=\beta^{*} d \theta_{P}$ being the pullback of the Liouville form of $T^{*} P$. Then $\beta$ is the symplectomorphism between $(T P, \dot{\omega})$ and $\left(T^{*} P, d \theta_{P}\right)$ and the triplet $\left(T P, \tau_{P}, \kappa\right)$, where $\tau_{P}: T P \longrightarrow P$ is the canonical projection, and $\kappa$ is the one-form such that $d \kappa=\dot{\omega}$, defines the cotangent bundle structure on $T P$.

Any Lagrangian submanifold of $(T P, \dot{\omega})$ is locally generated by the generating familyMorse family (cf. [20]) in the cotangent bundle structure $\left(T P, \tau_{P}, \kappa\right)$ on $T P$. In local terms it means that there is an open neighbourhood of every point of $D$ such that in Darboux coordinates around the chosen point, in which

$$
\kappa=\sum_{i=1}^{n}\left(\dot{y}_{i} d x_{i}-\dot{x}_{i} d y_{i}\right),
$$

the differential system $D$ is described by the following system of equations, $\kappa_{\mid D}=-d F$ :

$$
\begin{aligned}
\dot{x}_{i} & =\frac{\partial F}{\partial y_{i}}(x, y, \lambda), & & 1 \leq i \leq n, \\
\dot{y}_{j} & =-\frac{\partial F}{\partial x_{j}}(x, y, \lambda), & 1 & \leq j \leq n, \\
0 & =\frac{\partial F}{\partial \lambda_{k}}(x, y, \lambda), & & 1 \leq k \leq m,
\end{aligned}
$$

where $F: \mathbb{R}^{2 n} \times \mathbb{R}^{m} \longrightarrow \mathbb{R}$ is a smooth function in a neighbourhood $\mathcal{O}$ of zero in $\mathbb{R}^{2 n} \times \mathbb{R}^{m}$.

Definition 2.2. Let $(P, \omega)$ be a symplectic manifold. A first order differential system $D$ is called an implicit Lagrangian differential system if it is also a Lagrangian submanifold of the symplectic space $(T P, \dot{\omega})$. 
An implicit Lagrangian differential system is called an integrable Lagrangian differential system or integrable Lagrangian system (resp. weak integrable Lagrangian system) if it is integrable (resp. weak integrable).

When the number of Morse parameters is $m=0$, this means that $D$ is a graph of the function $(x, y) \mapsto\left(\frac{\partial F}{\partial y}(x, y),-\frac{\partial F}{\partial x}(x, y)\right)$ and the Hamiltonian field $\vec{F}$ of $F$ in $(P, \omega)$ is tangent to $D$ so $D$ is integrable. Moreover, we see immediately that any integral curve $D$ must be an integral curve of $\vec{F}$.

Let $D$ be an implicit Lagrangian differential system. Denote by $C_{D}$ the critical values of the projection $\left.\tau_{P}\right|_{D}: D \longrightarrow P$. The set $C_{D}$ will be called the differential (or Hamiltonian) caustic of $D$. Assume $D$ is integrable, then if $\gamma: I \longrightarrow P$ is an integral curve of $D$ passing through the point $\gamma(0)=p \in C_{D}$, then $\dot{\gamma}(0)$, belonging to $\Gamma_{D}$ - the set of critical points of the projection $\left.\tau_{P}\right|_{D}: D \longrightarrow P$, has to be tangent to $C_{D}$. Further on we assume that $C_{D}$ (and $\Gamma_{D}$ respectively) is a stratified set, i.e. there is a collection of smooth manifolds $C_{D}^{k}$ and $\Gamma_{D}^{k}, k \in J$, such that $C_{D}=\bigcup_{k \in J} C_{D}^{k}, \Gamma_{D}=\bigcup_{k \in J} \Gamma_{D}^{k}$. This assumption is fulfilled for the locally $\mathcal{A}$-finite maps (cf. [11]). Thus the tangency of $\gamma(0)$ to $C_{D}$ is understood as a tangency to the corresponding stratum of $C_{D}$. Let us define

$$
\Delta_{D}=\left\{v \in T D: T \tau_{P}(v)=\tau_{T P}(v), v \in T D\right\}
$$

then we have naturally $T \tau_{P}\left(\Delta_{D}\right) \subset D \subset T P$, and $T \tau_{P}\left(\Delta_{D} \cap T_{\Gamma_{D}} D\right) \subset T C_{D}$, where as we can easily check $T \tau_{P}\left(\Delta_{D} \cap T_{\Gamma_{D}} D\right)=\Gamma_{D}$. So the curve $\gamma$ whose tangent lift is passing through the point belonging to $\Gamma_{D}$, has to be tangent to the differential caustic point. Thus we have obtained the following necessary condition for integrability of $D$.

Proposition 2.1 (cf. [7]). Let $D$ be a Lagrangian differential system and suppose that its caustic $C_{D}$ is a stratified set, $C_{D}=\bigcup_{k \in J} C_{D}^{k}$. Then for all strata $C_{D}^{k}$ the tangent space $T C_{D}^{k}$ contains the corresponding stratum $\Gamma_{D}^{k}$ of the set of critical points of $\left.\tau_{P}\right|_{D}$.

3. Lagrangian manifolds and Pontryagin's maximum principle. Consider $q: S \longrightarrow X$ to be a locally trivial fibration of a manifold $S$ with a typical fibre manifold $U$ onto a manifold $X$ and denote by $n$ the dimension of $X$ and $m$ the dimension of the fibre. Suppose we have the map $\Gamma: S \longrightarrow T X$ (which is the vector field along $q$ ) such that $q=\tau_{X} \circ \Gamma$ if $\tau_{X}: T X \longrightarrow X$ is a canonical projection. An admissible curve or $S$-curve is an absolutely continuous map $\gamma: I \longrightarrow X$ such that $\dot{\gamma}(t)$ belongs to $\Gamma\left(S_{\gamma(t)}\right)$ for almost every $t \in I$, where $S_{\gamma(t)}$ is the fibre of $S$ over $\gamma(t)$. The manifold $U$ which is the "typical fibre" of $S$ is called the control space and $X$ is called the state space.

Take a Lagrangian function on $T X$, that is a differentiable map $L: T X \longrightarrow \mathbb{R}$. Given two fixed points $x_{0}$ and $x_{1}$ suppose that the set $\Omega_{x_{0}, x_{1}}$ of $S$-curves $\gamma:\left[t_{0}, t_{1}\right] \longrightarrow X$ such that $\gamma\left(t_{0}\right)=x_{0}$ and $\gamma\left(t_{1}\right)=x_{1}$ is not empty. Then we can consider the optimal problem of minimizing the functional

$$
\int_{t_{0}}^{t_{1}} L(\gamma(t), \dot{\gamma}(t)) d t
$$

on the set of $S$-curves belonging to $\Omega_{x_{0}, x_{1}}$. If there exists an $S$-curve which realizes this minimum we call it an optimal curve. A classical necessary condition for an $S$-curve to be optimal is given by the Pontryagin maximum principle. 
Before giving these conditions in terms of implicit Lagrangian differential system, we must introduce some notations. Denote by $T^{*} X \times_{X} S$ the bundle product over $X$ of the cotangent bundle $T^{*} X$ and $S$, and consider the map

$$
H: T^{*} X \times_{X} S \times \mathbb{R} \longrightarrow \mathbb{R}
$$

defined by $H(p, v, \nu)=\langle p, \Gamma v\rangle+\nu L(\Gamma v)$, where $\langle$,$\rangle is the duality bracket in T^{*} X$. This function is called classically the Hamiltonian of the optimal control problem. In local coordinates $\left(x_{i}, \dot{x}_{i}, y_{j}, \dot{y}_{j}, \lambda_{k}, \nu\right), 1 \leq i, j \leq n, 1 \leq k \leq m$, on $T T^{*} X \times_{X} S \times \mathbb{R}$, the map $\Gamma$ can be locally defined by the system of functions

$$
\dot{x}_{j}=f_{j}\left(x_{1}, \ldots, x_{n}, \lambda_{1}, \ldots, \lambda_{m}\right), \quad 1 \leq j \leq n,
$$

and we have

$$
H(x, y, \lambda, \nu)=\sum_{j=1}^{n} y_{j} f_{j}(x, \lambda)+\nu L(x, \lambda) .
$$

To this problem we can associate a one-parameter family of implicit Lagrangian differential systems $\left\{\mathcal{H}_{\nu}\right\}_{\nu \in \mathbb{R}}$ on $P=T^{*} X$, which is locally defined by:

$$
\begin{array}{rlrl}
\dot{x}_{i} & =\frac{\partial H}{\partial y_{i}}(x, y, \lambda, \nu), & & 1 \leq i \leq n, \\
\dot{y}_{j} & =-\frac{\partial H}{\partial x_{j}}(x, y, \lambda, \nu), & 1 \leq j \leq n, \\
0 & =\frac{\partial H}{\partial \lambda_{k}}(x, y, \lambda, \nu), & & 1 \leq k \leq m .
\end{array}
$$

With this notation, the classical Pontryagin maximum principle (cf. [17]) can be (incompletely) formulated in the following way:

THEOREM 3.1. If an $S$-curve of $\Omega_{x_{0}, x_{1}}$ defined on $I$, is optimal for the Lagrangian $L$, then there exists an absolutely continuous integral curve $(\gamma, p): I \longrightarrow \mathcal{H}_{\nu}$ for some $\nu \in \mathbb{R}$ such that if $\nu=0$ then we must have $p(t)$ non-zero for all $t \in I$.

REMARK 3.1. In the context of the previous theorem, we have even more, namely

$$
H(x(t), y(t), \lambda(t), \nu)=\text { const. }
$$

which is a supplementary condition in a complete version of the Pontryagin maximum principle. For absolutely continuous curve, this property is not true in general, for implicit Lagrangian differential system, but here this result depends strongly on the optimality of the $S$-curve.

Definition 3.1. An absolutely continuous integral curve $(\gamma, p): I \longrightarrow \mathcal{H}_{\nu}$ for some $\nu \in \mathbb{R}$ is called a bi-extremal and a field of the one-forms $p(t)$ along $\gamma$ is called an adjoint vector. This bi-extremal is called normal (resp. abnormal) bi-extremal if $\nu \neq 0$ (resp. $\nu=0$ and $p(t) \neq 0$ for all $t \in I)$.

REMARK 3.2.

1) From the linearity of $H$ with respect to the variable $y$, it is easy to see that if $(\gamma, p)$ is a bi-extremal, then for every non-zero $\nu \in \mathbb{R},(\gamma, \nu p)$ is also a bi-extremal. So in the family of Lagrangian manifolds $\left\{\mathcal{H}_{\nu}\right\}_{\nu \in \mathbb{R}}$, it is sufficient to consider only two manifolds $\mathcal{H}_{0}$ and $\mathcal{H}_{1}$. 
2) For a given $S$-curve we can have an adjoint vector $p$ for which $(\gamma, p)$ is a normal bi-extremal and an adjoint vector $p^{\prime}$ for which $\left(\gamma, p^{\prime}\right)$ is an abnormal bi-extremal. If all bi-extremals $(\gamma, p)$, associated to $\gamma$ are abnormal, we say that $\gamma$ is strictly abnormal.

A crucial problem in the optimal control theory is to decide if an optimal curve $\gamma$ can be strictly abnormal. In this case, it is clear that $\gamma$ is independent of the Lagrangian $L$. In the opposite case, when an optimal curve $\gamma$ is not strictly abnormal, in many general problems of optimal control, we have an adjoint vector $p$ such that $(\gamma, p)$ is an integral curve of some "canonical Hamiltonian field" on $P=T^{*} X$; so these curves are smooth. To detect strictly abnormal optimal curve, we must study the abnormal bi-extremals.

Consider a regular $C^{\infty}$ distribution $\triangle$ on a manifold $X$, that is a sub-bundle $\triangle$ of $T X$. If we take on $\triangle$ a Riemannian metric $g$, then we are fitting to the previous situation with $S=\triangle$ and $L=g$. Then we have a sub-Riemannian structure on $X$ and the optimal curves of this problem are exactly the minimizing geodesics in sub-Riemannian geometry. It is well known that in such situation there can exist strictly abnormal geodesics (cf. [12], [9], [13], [10]). The abnormal bi-extremal of this problem have another characterization in our context, which we present now.

Given a sub-Riemannian regular distribution on $X$. Let us denote by $\Omega_{x_{0}}^{k}$ the set of $\triangle$-curves or horizontal curves $\gamma$ defined on $[0,1]$ with an origin $\gamma(0)=x_{0}$ and which are of class $k$ ( $k=0$ for absolutely continuous, or $k$ non-zero integer for $H^{k}$ class). The set $\Omega_{x_{0}}^{k}$ has a structure of Banach manifold (this is even the Hilbert manifold for $k=2$ ). The map end : $\Omega_{x_{0}}^{k} \longrightarrow X$ defined by end $(\gamma)=\gamma(1)$ is smooth and when $\triangle \neq T X$, this map has always singular points. These singular points are exactly the curves $\gamma$ for which there exists an adjoint vector $p$ such that $(\gamma, p)$ is an abnormal bi-extremal (see [12], [14]). Precisely, in local coordinates, if $Z_{1}, \ldots, Z_{m}$ is a local basis of $\triangle$ then the horizontal curves are solutions of the system of differential equations

$$
\dot{x}(t)=\sum_{i=1}^{m} \lambda_{i}(t) Z_{i}(x(t))
$$

for some smooth functions $\lambda_{i}(t)$.

So the implicit Lagrangian system $D$ associated to the problem is locally generated by the Morse family

$$
\begin{array}{rlrl}
F: \mathbb{R}^{2 n} \times \mathbb{R}^{m} \longrightarrow \mathbb{R}, & F(x, y, \lambda)=\sum_{i=1}^{m} \lambda_{i}\left\langle y, Z_{i}\right\rangle \\
\dot{x}_{j}=\sum_{i=1}^{m} \lambda_{i} Z_{i j}(x), & 1 \leq j \leq n, \\
\dot{y}_{j}=-\sum_{i=1}^{m} \lambda_{i}\left\langle y, \frac{\partial Z_{i}}{\partial x_{j}}(x)\right\rangle, & 1 \leq j \leq n, \\
0=\left\langle y, Z_{i}(x)\right\rangle & & 1 \leq i \leq m,
\end{array}
$$

where $Z_{i}=\sum_{j=1}^{n} Z_{i j} \frac{\partial}{\partial x_{j}}$.

This implicit Lagrangian differential system is exactly the one which was previously denoted by $\mathcal{H}_{0}$ with $S=\triangle$ and $F$ is in fact the Hamiltonian of the control problem. 
4. Integrability conditions. In this section, we will prove the integrability conditions, when the generating Morse family is not too singular and also necessary and sufficient conditions for integrability in the context of some genericity assumptions.

Consider an ideal $\mathcal{I}$ in the ring $\mathcal{A}(U)$ of smooth functions defined in a neighbourhood $U$ of $(x, y, \lambda) \in \mathbb{R}^{2 n+m}$. We denote by $Z(\mathcal{I})$ the zero set of $\mathcal{I}$, that is the set of points $z \in U$ such that $f(z)=0$ for each $f \in \mathcal{I}$. Recall that $\mathcal{I}$ is principal if $\mathcal{I}$ is generated by single function and is said to have the property of zeros if each smooth function $f$ vanishing on $Z$ belongs to $\mathcal{I}$ (cf. [15]). Let $D$ be a Lagrangian submanifold of $T P$ and consider a generating Morse family $F$ defined on a neighbourhood $U$ of $\mathbb{R}^{2 n} \times \mathbb{R}^{m}$. We denote by $S_{F}(x, y, \lambda)$ the field on $U$ of symmetric matrices

$$
\left(\frac{\partial^{2} F}{\partial \lambda_{i} \partial \lambda_{j}}(x, y, \lambda)\right) \text {. }
$$

Let $\Sigma_{r}$ be the set of points at which the rank of $S_{F}(x, y, \lambda)$ is $r$ and $\Sigma=\bigcup_{r=0}^{m-1} \Sigma_{r}$ be the singular set of $S_{F}(x, y, \lambda)$. Finally, we denote by $\{f, g\}$ the Poisson bracket on $\mathbb{R}^{2 n}$ for the canonical symplectic structure.

THEOREM 4.1. Let $D$ be a Lagrangian submanifold of $(T P, \dot{\omega})$, and suppose that there exists, on a connected open set $U$ of $\mathbb{R}^{2 n} \times \mathbb{R}^{m}$, a generating Morse family $F$ defined on $U$ such that:

(i) $\Sigma_{m}$ is not empty on $U$ and locally around each point of $\Sigma$ the ideal $\mathcal{I}$ generated by $\operatorname{det} S_{F}$ possesses the property of zeros;

(ii) if $M_{F}$ is the $m \times(m+1)$ matrix obtained from the column vectors of $S_{F}$ and the column vector $\left\{\frac{\partial F}{\partial \lambda}, F\right\}$, and $\Sigma_{m-1}$ is not empty, the rank of $M_{F}$ is $m-1$ on a dense set of $\Sigma_{m-1}$.

If these two conditions are satisfied, then $\Sigma$ contains a hypersurface which is an open dense set of $\Sigma$. Moreover, on the subset

$$
\mathcal{O}=\left\{\left(\frac{\partial F}{\partial x}(x, y, \lambda),-\frac{\partial F}{\partial y}(x, y, \lambda), x, y\right): \frac{\partial F}{\partial \lambda}(x, y, \lambda)=0, \quad(x, y, \lambda) \in U\right\},
$$

there exists a unique (Hamiltonian) vector field $\vec{H}$ such that for any $v \in \mathcal{O}$ the integral curve of $\vec{H}, t \mapsto(\gamma(t), \dot{\gamma}(t))$ is the unique integral curve of $D$ at $v$. In particular, if these hypotheses are fulfilled for each open covering of $D$, then $D$ is a Lagrangian system and moreover, there exists a unique vector field $Z$ such that each integral curve of $Z$ is an integral curve of $D$. On the complement of the singular set $C_{D}$ of the mapping $\left.\tau_{P}\right|_{D}$ such a curve is unique.

REMARK 4.1. If condition (i) is fulfilled for each open covering of $D$, from the density of $\Sigma_{m}$ it is easy to see that condition (ii) is then a necessary and sufficient condition for integrability (cf. [7]).

By $M_{s}$ we denote the set of symmetric $m \times m$ matrices over $\mathbb{R}$. The family of subsets of matrices of rank $r\left\{\Sigma_{r}\right\}_{r \in \mathbf{N}}$ defines a stratification of $M_{s}$. The codimension of the stratum $\Sigma_{r}$ is

$$
\frac{(m-r)(m-r+1)}{2}
$$


For a Lagrangian submanifold $D$ of $(T P, \dot{\omega})$, we have given a generating Morse family $F$ on an open set $U \subset \mathbb{R}^{2 n} \times \mathbb{R}^{m}$. The matrix $S_{F}$ is a map from $U$ to $M_{s}$. If this map is transverse to the stratification, then the function $\operatorname{det} S_{F}$ possesses the property of zeros ([16]). Moreover, the transversality condition implies that if the image of $S_{F}$ meets a stratum $\Sigma_{r}$ for $r \leq m-1$ then this image intersects all strata $\Sigma_{r}^{\prime}$ for $r \leq r^{\prime} \leq m$. From Theorem 4.1 and Remark 4.1, we obtain:

Corollary 4.1. Consider a Lagrangian submanifold $D$ of $(T P, \dot{\omega})$ and suppose that for every point in $D$ there exists a generating Morse family $F: U \subset \mathbb{R}^{2 n} \times \mathbb{R}^{m} \longrightarrow \mathbb{R}$ such that the associated matrix $S_{F}$ is transverse to the stratification $\left\{\Sigma_{r}\right\}_{r \in \mathbf{N}}$ in $M_{s}$. $A$ necessary and sufficient condition for integrability of $D$ is:

On the stratum $\Sigma_{m-1}$ the vector column $\left\{\frac{\partial F}{\partial \lambda}, F\right\}$ is linearly dependent on the vector columns of the matrix $S_{F}$.

When the field of matrices $S_{F}$ is transverse to the stratification $\left\{\Sigma_{r}\right\}_{r \in \mathbf{N}}$ in $M_{s}$ we will say that $S_{F}$ is a generic field of matrices. Now we present a result about integrability which is not "generic" in the previous sense.

TheOREM 4.2. Let $D$ be a Lagrangian manifold of $(T P, \dot{\omega})$ and suppose that $D$ has, for every point of $D$, a generating Morse family $F$ defined on an open neighbourhood $U$ of $\mathbb{R}^{2 n} \times \mathbb{R}^{m}$ such that at each point of $U$ the rank of the matrix $S_{F}$ is $r<m$ everywhere, and suppose that $\left\{\frac{\partial F}{\partial \lambda}, F\right\}$ is a linear combination of the vector columns of $S_{F}$ everywhere. Then $D$ is a Lagrangian differential system. Moreover, the integral curve at each point of $D$ is not unique.

For the proof of Theorems 4.1 and 4.2 we use the following lemma.

LEMMA 4.1. Suppose that there exists a $C^{\infty}$ solution $\mu(x, y, \lambda)$ on an open set $U \subset \mathbb{R}^{2 n} \times \mathbb{R}^{m}$ of the implicit equation

$$
S_{F} \mu=\left\{\frac{\partial F}{\partial \lambda}, F\right\}
$$

then $D$ is integrable at each point of

$$
\left\{\left(\frac{\partial F}{\partial y}(x, y, \lambda),-\frac{\partial F}{\partial x}(x, y, \lambda), x, y\right): \frac{\partial F}{\partial \lambda}(x, y, \lambda)=0\right\}
$$

for each $(x, y, \lambda) \in U$.

Proof. Suppose that $t \mapsto(x(t), y(t), \lambda(t))$ is a smooth solution of (1). By differentiation of

$$
\frac{\partial F}{\partial \lambda_{k}}(x(t), y(t), \lambda(t))=0
$$

with respect to $t$ we obtain an implicit equation for $\mu \in \mathbb{R}^{m}$

$$
0=\frac{\partial^{2} F}{\partial \lambda_{k} \partial \lambda_{i}}(x, y, \lambda) \mu_{i}-\frac{\partial^{2} F}{\partial \lambda_{k} \partial x_{j}} \frac{\partial F}{\partial y_{j}}(x, y, \lambda)+\frac{\partial^{2} F}{\partial \lambda_{k} \partial y_{i}} \frac{\partial F}{\partial x_{i}}(x, y, \lambda) .
$$

This expression can be written in the following way, using the Poisson bracket and the previous notation:

$$
S_{F} \mu=\left\{\frac{\partial F}{\partial \lambda}, F\right\}
$$


so, if we can find a smooth solution $\mu(x, y, \lambda)$ of the system $(6)$, then the system of ordinary differential equations

$$
\begin{array}{ll}
\dot{x}_{i}=\frac{\partial F}{\partial y_{i}}(x, y, \lambda), & 1 \leq i \leq n, \\
\dot{y}_{j}=-\frac{\partial F}{\partial x_{j}}(x, y, \lambda), & 1 \leq j \leq n, \\
\dot{\lambda}_{k}=\mu_{k}(x, y, \lambda), & 1 \leq k \leq m,
\end{array}
$$

is locally integrable.

Proof of Theorem 4.2. As the matrix $S_{F}$ is symmetric then by choosing any scalar product $\langle$,$\rangle on \mathbb{R}^{m}$ we can consider $S_{F}$ as a symmetric operator on this space. Now from the fact of constant rank $r<m$ and the symmetry of $S_{F}$ there exist subbundles $E_{1}$ and $E_{2}$ of the trivial bundle $E=U \times \mathbb{R}^{m}$ such that they are $S_{F}$ invariants $E_{1}=\operatorname{Im} S_{F}$ and $E_{2}=\operatorname{Ker} S_{F}$ and $E=E_{1} \oplus E_{2}$. Now $\left\{\frac{\partial F}{\partial \lambda}, F\right\}$ belongs to $E_{1}$ everywhere and the restriction of $S_{F}$ to $E_{1}$ is invertible. Take a local orthonormal basis $\left(Z_{1}, \ldots, Z_{r}\right)$ of $E_{1}$ and $\left(Z_{r+1}, \ldots, Z_{m}\right)$ orthonormal of $E_{2}$. Denote by $C$ the column matrix of the components of $\left\{\frac{\partial F}{\partial \lambda}, F\right\}$ on the previous basis. Of course these components are 0 on $\left(Z_{r+1}, \ldots, Z_{m}\right)$. Let $P$ be the orthogonal matrix field of components of this local basis. Putting $S^{\prime}=P^{-1} S_{F} P$ we can solve $S X=C$. Moreover, take any section $K$ of the kernel bundle $E_{2}$ and denote also by $K$ the column matrix associated. Then we have $A(X+K)=C$ too. Then $\mu=P^{-1}(X+K)$ is a $C^{\infty}$ solution of (5) and from Lemma 4.1, $D$ is locally integrable. As $r<m$, the choice of $K$ may be arbitrary and so we do not have the uniqueness of the local integral curves.

Proof of Theorem 4.1. By using Lemma 4.1 it remains to show that, under conditions (i) and (ii), the system (5) has always a smooth solution $\mu(x, y, \lambda)$. The function which prescribes to $z$ the rank of $S_{F}(z)$ is lower semi-continuous. As $U$ is convex, then the subset of $\Sigma_{m}$, at which $S_{F}$ is of maximal rank $m$, is an open dense set in $U$. Moreover, by the same argument of lower semi-continuity, the closure of $\Sigma_{m-1}$ (if this set is not empty), is the union of all sets $\Sigma_{r}$ for $r<m$.

LEMMA 4.2. Let $S$ be a $C^{\infty}$ field of symmetric matrices of order $m$ on a neighbourhood $V$ of $0 \in \mathbb{R}^{N}$ and of rank $m-1$ at 0 . Suppose that $\mathcal{I}$, the ideal generated by $\operatorname{det} S$, is not locally trivial and possesses the property of zeros. Then there is an open dense set of $\Sigma$ which is a codimension one submanifold. Moreover, if $C$ is a smooth matrix column of dimension $m$ on $V$ such that $C$ is linearly dependent on the vectors of $S$ on a dense subset of $\Sigma$, then the equation $S X=C$ has a $C^{\infty}$ unique smooth solution on $V$.

From Lemma 4.2 we conclude that if $\Sigma_{m-1}$ is not empty, it contains an open dense submanifold. So again from the Lemma $4.2, S_{F}$ is invertible on the open dense set $\Sigma_{m}$. So the system (5) is a Cramer system. In particular, if $S_{F}^{*}$ is the $C^{\infty}$ field of the matrix of co-factor of $S_{F}$, we have

$$
\operatorname{det}\left(S_{F}\right) \mu=S_{F}^{*}\left\{\frac{\partial F}{\partial \lambda}, F\right\} .
$$

Using the hypothesis (ii), from the Lemma we know that we can extend the solution 
of (5) onto $\Sigma_{m-1}$. Consequently, the terms of the matrix $S_{F}^{*}\left\{\frac{\partial F}{\partial \lambda}, F\right\}$ can be divided by $\operatorname{det} S_{F}$ on $\Sigma_{m-1}$ (cf. [7]). As $\Sigma$ is the closure of $\Sigma_{m-1}$, it follows that all terms of $S_{F}^{*}\left\{\frac{\partial F}{\partial \lambda}, F\right\}$ are zero on the closure of $\Sigma$ so again by condition (i) we can conclude that they are divisible by $\operatorname{det} S_{F}$ and so we obtain a unique $C^{\infty}$ solution $\mu$.

Now, around each point $\left(x_{0}, y_{0}, \lambda_{0}\right) \in \Sigma_{m}$, by the implicit function theorem, there exists a smooth map $v(x, y)$ such that

$$
\frac{\partial F}{\partial \lambda}(x, y, v(x, y))=0
$$

and $v\left(x_{0}, y_{0}\right)=\lambda_{0}$; moreover, by differentiation we have

$$
\left(\frac{\partial v}{\partial x}(x, y), \frac{\partial v}{\partial y}(x, y)\right)=\left(S_{F}\right)^{-1}\left\{\frac{\partial F}{\partial \lambda}, F\right\}=\mu(x, y, v(x, y))
$$

so $v$ is uniquely defined. By local uniqueness we can construct on $\Sigma_{m}$ a smooth functionagain denoted by $v$-such that (7) and (8) are satisfied. This means that over $\Sigma_{m}, D$ is the graph of the function

$$
(x, y) \mapsto\left(\frac{\partial F}{\partial y}(x, y, v(x, y)),-\frac{\partial F}{\partial x}(x, y, v(x, y))\right)
$$

and so the solution of the differential system (10) are integral curves of the Hamiltonian field of $F(x, y, v(x, y))$. So this vector field can be extended on all $U$. The complement of the critical set $C_{D}$ is the graph of a closed one-form $\delta$ over an open set of $P$. So from the previous argument, this vector field can be extended to a vector field on $D$.

Proof of Lemma 4.2. Let $\langle$,$\rangle be the canonical scalar product on \mathbb{R}^{k}$. Then we can consider $S$ as a linear operator which is symmetric relative to $\langle$,$\rangle . Consider the$ characteristic polynomial $P(z, t)=\operatorname{det}(S-t \mathrm{Id})$ of $S$. As $S$ is symmetric, all roots of $P(z, t)$ are real numbers. From the hypothesis, we have $P(0,0)=0$ but $\frac{\partial P}{\partial t}(0,0) \neq 0$. By the implicit function theorem, there exists a neighbourhood $V^{\prime}$ of $0 \in \mathbb{R}^{N}$ and a $C^{\infty}$ function $\phi: V^{\prime} \longrightarrow \mathbb{R}$ such that $\phi(0)=0$ and $P(z, \phi(z))=0$ on $V^{\prime}$. In fact, the set $\left\{z \in V^{\prime}: \phi(z)=0\right\}$ is exactly the set $\Sigma$ of points at which the rank of $S$ is $m-1$. Suppose that $\Sigma$ has a non-empty interior. Then on a small enough neighbourhood of a point $z \in \Sigma$, the rank of $S$ would be $m-1$ and then $\operatorname{det} S$ would be identically zero on this neighbourhood and the ideal $\mathcal{I}$ would be trivial on this neighbourhood which contradicts our hypothesis. So, the complement $\Sigma_{m}$ of $\Sigma$ in $V^{\prime}$ is dense.

Now we show that there exists an open dense subset $\hat{\Sigma}$ of $\Sigma$ which is a $C^{\infty}$ hypersurface. Suppose that there exists an open set of $\Sigma$ such that $d \phi=0$. Without loss of generality, we can suppose that it is a neighbourhood of $0 \in \Sigma$. Then it follows that for all partial derivatives $\frac{\partial \phi}{\partial z_{i}}=0$ on this open set for all $1 \leq i \leq N$. So by the property of zeros, we have $\frac{\partial \phi}{\partial z_{i}}=f_{i} \phi$. As $\phi(0)=0$, this imposes by induction on $i=1, \ldots, N$ that $\phi$ does not depend of each variable $\left(z_{1}, \ldots, z_{i}\right)$ and finally that $\phi$ is identically zero which is again a contradiction with the non-triviality of $\mathcal{I}$.

We can write $P(z, t)=(t-\phi(z)) P_{1}(z, t)$ with $P_{1}(0, t) \neq 0$. So on $\Sigma_{m}$ the set

$$
E_{1}=\left\{(z, v) \in\left\{V-\Sigma^{\prime}\right\} \times \mathbb{R}^{k}: S v=\phi(z) v\right\}
$$


is a one-dimensional bundle. By using the symmetry of $S$, on $\Sigma_{m}$, we can decompose the trivial bundle $E=V^{\prime} \times \mathbb{R}^{k}$ in a Whitney sum $E_{1} \oplus E_{2}$ such that $E_{2}$ is an $(m-1)$ dimensional bundle invariant for $S$ and $S$ is invertible in restriction to $E_{2}$. On the other hand, on $\Sigma$, at each point we have the unique decomposition $\mathbb{R}^{k}=\operatorname{Ker} S \oplus \operatorname{Im} S$. As $\Sigma^{\prime}$ is dense in $V^{\prime}$ and $E_{1}^{\prime}=E_{1} \oplus K$ is also invariant by $S$, by passing to the limit in an adequate Grassmannian, for all $z_{0} \in \Sigma$, we have

$$
\lim _{z \rightarrow z_{0}} E_{2}=\operatorname{Im} S_{z_{0}}, \quad \lim _{z \rightarrow z_{0}}\left(E_{1} \oplus K\right)=\operatorname{Ker} S_{z_{0}} .
$$

Now we take a $C^{\infty}$ field $C$ on $V$ of $m$-dimensional column matrices such that $C$ is everywhere linearly dependent on the set of the column matrices of $S$ on a dense set of $\Sigma$ and consequently on all $\Sigma$ by continuity. As the map $z \mapsto \operatorname{Ker} S_{z}$ and $z \mapsto \operatorname{Im} S_{z}$ are continuous (as functions in an adequate Grassmannian), from (7) we deduce that there exists a field $P$ of invertible matrices of order $k$, of class $C^{0}$, such that $S^{\prime}=P^{-1} S P$ is of the type

$$
\left(\begin{array}{cc}
\phi & 0 \\
0 & S^{\prime}
\end{array}\right)
$$

Consider the field $C^{\prime}=P^{-1} C P$. From the hypothesis, it follows that

$$
C^{\prime}=\left(\begin{array}{c}
f \phi \\
C^{\prime}
\end{array}\right)
$$

where $f$ is a continuous function. So the solution $X^{\prime}$ of $S^{\prime} X^{\prime}=C^{\prime}$ is equal to

$$
X=\left(\begin{array}{c}
f \\
\left(S^{\prime}\right)^{-1} C^{\prime}
\end{array}\right)
$$

It follows that we can extend the solution of $S X=C$ on $\Sigma$.

Question. We have seen that, under the hypotheses of Theorem 4.1, there exists a smooth integral curve at every point of $D$ and this curve is unique on the complement of the singular set $C_{D}$ of $\left.\tau_{P}\right|_{D}$. Can it happen that the integral curve at a point of $C_{D}$ may not be unique?

EXAMPLE 4.1. For a given integer $n \geq 1$ choose $m$ such that $\frac{m(m+1)}{2} \leq n$. Choose an injection $\sigma$ of the $\{(i, j): 1 \leq i \leq j \leq m\}$ into the set of integers $\{1,2, \ldots, n\}$ such that $\sigma_{11}=1$ and denote by $J$ the set of values $\sigma_{i j}$. Consider the implicit Lagrangian differential system generated by the function $F$ defined by

$$
F(x, y, \lambda)=\sum_{i, j=1}^{m} \phi_{i j} \lambda_{i} \lambda_{j}+\sum_{l \notin J} x_{l} y_{l}
$$

with $\phi_{11}(x, y)=\sum_{1 \leq i \leq j \leq m}^{n} x_{\sigma_{i j}} y_{\sigma_{i j}}$, and otherwise $\phi_{i j}(x, y)=\phi_{j i}(x, y)=x_{\sigma_{i j}}$. The matrix $S_{F}$ is $\left(\phi_{i j}\right)$ which is transverse to the stratification $\left\{\Sigma_{r}\right\}_{r=0, \ldots, m}$ of $M_{s}$ on the open set $\mathcal{O}=\left\{(x, y) \in \mathbb{R}^{2 n} \times \mathbb{R}^{m}:(x, y) \neq(0,0)\right\}$. Moreover, it is easy to see that the ideal generated by $\operatorname{det} S_{F}$ possesses (locally) the property of zeros on $\mathbb{R}^{2 n} \times \mathbb{R}^{m}$. As

$$
\left\{\phi_{11}, \phi_{i j}\right\}=\phi_{i j}
$$


for $(i, j) \neq(1,1)$ and 0 otherwise, and also

$$
\left\{\phi_{i j}, \phi_{k l}\right\}=0
$$

for $(i, j)$ and $(k, l)$ different from $(1,1)$, we conclude that

$$
\left\{\frac{\partial F}{\partial \lambda}, F\right\}
$$

is a linear combination of the vector columns of $S_{F}, D$ is a Lagrangian differential system (i.e. integrable) on $\mathcal{O}$ from Corollary 4.1 and on $\mathbb{R}^{2 n} \times \mathbb{R}^{m}$ from Theorem 4.1.

ExAmple 4.2. Consider a distribution $\triangle$ on an $n$-dimensional manifold $X$. We consider the control problem for curves, which are tangent to $\triangle$ almost everywhere. Consider any Lagrangian $L$ on $D$. Then to this problem one associates naturally two implicit Lagrangian differential systems $D_{0}$ and $D_{1}$, locally defined in the following way (see Section 3):

Consider a local basis $\left(Z_{1}, \ldots, Z_{m}\right)$ of $\triangle$. A curve $\gamma: I \longrightarrow X$ is horizontal if we have

$$
\dot{\gamma}(t)=\sum_{i=1}^{m} \lambda_{i}(t) Z_{i}(\gamma(t))
$$

Then the generating Morse families for $D_{\nu}, \nu=0,1$, are $F^{\nu}: \mathbb{R}^{2 n} \times \mathbb{R}^{m} \longrightarrow \mathbb{R}$ defined by

$$
F^{\nu}(x, y, \lambda)=\left\langle y, \sum_{i=1}^{m} \lambda_{i} Z_{i}(x)\right\rangle+\nu L(x, \lambda) .
$$

- For $\nu=0$, the integral curves of $D_{0}$ give rise to abnormal bi-extremals. If $F$ is linear in the variables $\lambda$, we cannot apply the theorem. The projection of bi-extremals are the singular curves of the distribution. Such curves are solutions of the system of linear differential equations (cf. [7]):

$$
\sum_{i=1}^{m} \lambda_{i}\left\langle y,\left[Z_{i}, Z_{j}\right]\right\rangle=0
$$

for non-trivial $\lambda$. In fact it corresponds to the existence of a non-zero curve $\lambda$ such that

$$
\left\{\frac{\partial F}{\partial \lambda_{j}}, F\right\}=0, \quad 1 \leq j \leq m
$$

or in equivalent form,

$$
\sum_{i=1}^{m} \lambda_{j}\left\{H_{i}, H_{j}\right\}=0, \quad 1 \leq j \leq m
$$

where $H_{i}(x, y)=\left\langle y, Z_{i}(x)\right\rangle$.

If the dimension $m$ of $\triangle$ is even, then the matrix $\left(\left\{H_{i}, H_{j}\right\}\right)$ has a non-zero kernel. So on the open set of points at which rank of the matrix is maximal we have non-trivial solutions of (13) and then by Theorem $4.2, D_{0}$ is integrable on this set, but in general the solutions are not unique. If $D_{0}$ is integrable then the conormal bundle $A_{\triangle} \subset T^{*} X$ is an isotropic submanifold of $D_{0}$ and all singular curves are tangent to the coisotropic distribution $\mathcal{C}=T A_{D} \cap\left[T A_{D}\right]^{\perp}$, where $\left[T A_{D}\right]^{\perp}$ is the symplectic orthogonal of $T A_{D}$.

When $m$ is odd, for generic distribution $\triangle$ the system (5) has only non-trivial solutions on the hypersurface $\Sigma$ whose equation is $H_{0}(x, y)=\operatorname{det}\left\{H_{i}, H_{j}\right\}=0$. 
- For $\nu=1$, the symmetric matrix associated to $F^{1}$ is

$$
\left(\frac{\partial^{2} L}{\partial \lambda_{i} \partial \lambda_{j}}(x, y, \lambda)\right)
$$

If we choose $L$ so that $S_{F}$ is transverse to the stratification of $M_{s}$ the equation of the first stratum $\Sigma_{m-1}$ is given by $\operatorname{det}\left(\frac{\partial^{2} L}{\partial \lambda_{i} \partial \lambda_{j}}(x, y, \lambda)\right)=0$ and the condition for integrability from Corollary 4.1 is

$$
\operatorname{rank}\left(\frac{\partial^{2} L}{\partial \lambda_{i} \partial \lambda_{j}}(x, \lambda), \sum_{i=1}^{m} \lambda_{j}\left[\left\{H_{i}, H_{j}\right\}-\frac{\partial^{2} L}{\partial \lambda_{i} \partial x_{j}} Z_{j j}\right]\right)=m-1
$$

on $\Sigma_{m-1}$. For instance if $L(x, \lambda)=\sum_{k, l=1}^{m} g_{k l} \lambda_{k} \lambda_{l}$ where $G=\left(g_{k l}\right)$ is a symmetric matrix, the first condition imposes the transversality to the stratification of $M_{s}$ and the last one is

$$
\operatorname{rank}\left(g_{k l}, \sum_{l=1}^{m} \lambda_{l}\left[\left\{H_{k}, H_{l}\right\}-\frac{\partial g_{k l}}{\partial x_{j}} Z_{i l}\right]\right)=m-1
$$

Unfortunately, for $m>1$, if $\triangle$ is not involutive, in general this Lagrangian differential system is not integrable if $\Sigma_{m-1}$ is not empty: the condition (ii) of Theorem 4.1 is satisfied only on the measure zero subset of $\Sigma_{m-1}$.

5. Equivalence and classification of implicit Lagrangian differential systems. Let $\left(D_{1}, p_{1}\right),\left(D_{2}, p_{2}\right)$ be two germs of implicit Lagrangian differential systems in $T P$. We assume $D_{1}, D_{2}$ are integrable systems. By an equivalence of the differential systems we call the symplectomorphism preserving integrability.

$$
\dot{\Phi}:(T P, d \kappa) \longrightarrow(T P, d \kappa)
$$

such that the following diagram commutes

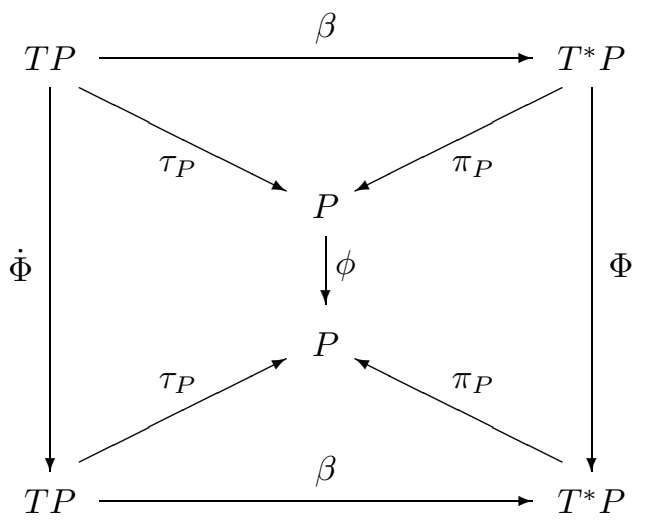

where $\Phi$ is a germ of a symplectomorphism of the cotangent bundle $\left(T^{*} P, d \theta_{P}\right)$ and $\phi$ is a germ of a symplectomorphism of $(P, \omega)$. 
Definition 5.1. We say that $\left(D_{1}, p_{1}\right),\left(D_{2}, p_{2}\right)$ are equivalent if there exists a germ of an equivalence $\dot{\Phi}$ such that

$$
\dot{\Phi}\left(D_{1}\right)=D_{2}, \quad \dot{\Phi}\left(p_{1}\right)=\dot{\Phi}\left(p_{2}\right) .
$$

We see that $\dot{\Phi}$ is the pull-back by $\beta$ of the standard equivalence $\Phi$ of a cotangent bundle $T^{*} P . \Phi$ is given by a symplectic lifting of $\phi, T^{*} \phi$ (cf. [2], [20]) and gradient of a smooth function $f$ on $P$. We easily see that the integrability of $D$ is preserved by the equivalences, which are symplectic liftings of a diffeomorphism $\phi$. However it is not so for the gradient additive equivalence.

Assume that $\Phi$ is the symplectomorphism

$$
\Phi(\bar{x}, \bar{y}, x, y)=\left(\bar{x}+\frac{\partial f}{\partial x}(x, y), \bar{y}+\frac{\partial f}{\partial y}(x, y), x, y\right)
$$

and the corresponding $\dot{\Phi}=\beta^{-1} \circ \Phi \circ \beta$ is defined by the differential $d f$ of a smooth function $f: P \longrightarrow \mathbb{R}$. Then we have the following lemma.

LEMma 5.1. If the implicit Lagrangian differential system $D$ is integrable, then the equivalent differential system $\Phi(D)$ is integrable if and only if

$$
\left.\beta^{-1} d f\right|_{C_{D}} \subset T C_{D} .
$$

Proof. If $F: \mathbb{R}^{2 n} \times \mathbb{R}^{m} \longrightarrow \mathbb{R}$ is a local generating family for $D \subset T P$, then the generating family for an equivalent system $\tilde{D}=\dot{\Phi}(D)$ has the form (cf. [2])

$$
F(\phi(x, y), \lambda)+f(x, y)
$$

where $\phi: \mathbb{R}^{2 n} \longrightarrow \mathbb{R}^{2 n}$ is a symplectomorphism-germ and $f: \mathbb{R}^{2 n} \longrightarrow \mathbb{R}$ is a smooth function-germ. If $D$ is integrable and $\gamma: I \longrightarrow \mathbb{R}^{2 n}$ is an integral curve through $\gamma(0) \in C_{D}$, where $\dot{\gamma}(0) \in \Gamma_{D}$, then $\tilde{D}$ is integrable with the integral curve $\tilde{\gamma}: I \longrightarrow \mathbb{R}^{2 n}$, through $\tilde{\gamma}(0) \in \phi^{-1}\left(C_{D}\right)$, with $\dot{\tilde{\gamma}}(0) \in \Gamma_{\tilde{D}}$ because of the tangency condition $\Gamma_{\tilde{D}} \subset$ $T \phi^{-1}\left(C_{D}\right)$. This condition follows from the assumption that $\beta^{-1} d f$ is a logarithmic vector field tangent to $C_{D}$.

Let the germ $(D, p)$ be generated by a Morse family-germ $G: P \times \mathbb{R}^{m} \longrightarrow \mathbb{R}$ such that $D$ is given by the equations

$$
\begin{array}{rlrl}
\dot{x}_{i} & =\frac{\partial G}{\partial y_{i}}(x, y, \lambda), & & 1 \leq i \leq n, \\
\dot{y}_{j} & =-\frac{\partial G}{\partial x_{j}}(x, y, \lambda), & 1 \leq j \leq n, \\
0 & =\frac{\partial G}{\partial \lambda_{k}}(x, y, \lambda), & 1 \leq k \leq m .
\end{array}
$$

By $W$ we denote the critical set

$$
W=\left\{(x, y, \lambda): F_{k}(x, y, \lambda):=\frac{\partial G}{\partial \lambda_{k}}(x, y, \lambda)=0\right\}
$$

and $\pi_{W}$ is the canonical projection, $\pi_{W}: W \ni(x, y, \lambda) \mapsto(x, y)$.

TheOREM 5.1. If the projection $\pi_{W}$ of the smooth hypersurface $W$ into $P$ has the Whitney singularities of type $\Sigma^{1}, \Sigma^{01}, \Sigma^{001}$ at 0 , then the germ of Lagrangian differential 
system $(D, 0)$ can be reduced, by the formal equivalence $\dot{\Phi}$, to one from the following normal forms:

$$
\begin{gathered}
G(x, y, \lambda)=\frac{1}{3} \lambda^{3}+y_{1} \lambda, \\
G(x, y, \lambda)=\frac{1}{4} \lambda^{4}+\frac{1}{2} y_{1} \lambda^{2}+x_{1} \lambda-\frac{1}{12} y_{1}^{2}, \\
G(x, y, \lambda)=\frac{1}{5} \lambda^{5}+\frac{1}{3} y_{1} \lambda^{3}+\frac{1}{2} x_{2} \lambda^{2}+y_{2} \lambda+\frac{1}{2} x_{1} .
\end{gathered}
$$

Proof. For Whitney's singularities of the projection $\pi_{W}$, the kernel of this projection is one-dimensional. Then by the reduction procedure of Morse parameters (generalized Morse Lemma [2]) we get the generating family with $m=1$ - one Morse parameter. For generic Whitney's singularities $W=\{(x, y, \lambda): F(x, y, \lambda)=0\}$ is a smooth hypersurface (cf. [11]). Now we consider $W$ as a hypersurface in the coisotropic hypersurface $Y=\{\mu=0\}$, which is contained in the extended symplectic space $\tilde{P}=T^{*} \mathbb{R} \times P$, endowed with the symplectic structure $\tilde{\omega}=d \mu \wedge d \lambda+\omega$. By an assumption the coisotropic projection $\pi: Y \longrightarrow P$, restricted to the hypersurface $W$, generically can have only Whitney's corank 1 singularities $\Sigma$. Now, by Arnold-Darboux Theorem (cf. [1], Th. 3, p. 7) we obtain three formal normal forms, according to the formal $\mathcal{R}_{+}$-equivalence of generating families for the projection $\pi_{W}$.

$$
F=\lambda^{2}+y_{1}, \quad F=\lambda^{3}+y_{1} \lambda+x_{1}, \quad F=\lambda^{4}+y_{1} \lambda^{2}+x_{2} \lambda+y_{2} .
$$

Thus the generating family $G$, with respect to the equivalence preserving integrability, can be written in three corresponding forms

$$
\begin{aligned}
\Sigma^{1}: G(x, y, \lambda) & =\frac{1}{3} \lambda^{3}+y_{1} \lambda+Q_{1}(x, y), \\
\Sigma^{01}: G(x, y, \lambda) & =\frac{1}{4} \lambda^{4}+\frac{1}{2} y_{1} \lambda^{2}+x_{1} \lambda+Q_{2}(x, y), \\
\Sigma^{001}: G(x, y, \lambda) & =\frac{1}{5} \lambda^{5}+\frac{1}{3} y_{1} \lambda^{3}+\frac{1}{2} x_{2} \lambda^{2}+y_{2} \lambda+Q_{3}(x, y) .
\end{aligned}
$$

By the calculation on the basis of the integrability condition (15) we get

$$
\begin{gathered}
Q_{1}(x, y) \in I\left(C_{D_{1}}\right), \\
Q_{2}(x, y)=-\frac{y_{1}^{2}}{12}+I\left(C_{D_{2}}\right), \\
Q_{3}(x, y)=\frac{1}{2} x_{1}+I\left(C_{D_{3}}\right),
\end{gathered}
$$

where $I\left(C_{D_{i}}\right)$ is the ideal of functions vanishing on $C_{D_{i}}$. In the corresponding cases we have $I\left(C_{D_{1}}\right)=\left\langle y_{1}\right\rangle, I\left(C_{D_{2}}\right)=\left\langle\left(y_{1} / 3\right)^{3}+\left(x_{1} / 2\right)^{2}\right\rangle$ and

$$
I\left(C_{D_{3}}\right)=\left\langle 16 y_{1}^{4} y_{2}-4 y_{1}^{3} x_{2}^{2}-128 y_{1}^{2} y_{2}^{2}+144 y_{1} x_{2}^{2} y_{2}+256 y_{2}^{3}-27 x_{2}^{4}\right\rangle .
$$

Thus choosing the simplest representants we obtain the desired normal forms of the theorem.

REMARK 5.1. If, instead of symplectomorphism $\phi$, in the equivalence of Lagrangian differential systems we take a general diffeomorphism, then especially in two-dimensional case of $P$ (cf. [3]), we get immediately the local classification of Lagrangian projections, 
and corresponding caustics. However in general case we lose the Hamiltonian structure and such equivalence is not distinguishing between Hamiltonian and general differential systems.

\section{References}

[1] V. I. Arnol'd, Lagrangian submanifolds with singularities, asymptotic rays and the open swallowtail, Funktsional. Anal. i Prilozhen. 15:4 (1981), 1-14 (Russian); English transl.: Functional Anal. Appl. 15 (1981), 235-246.

[2] V. I. Arnol'd, S. M. Guseı̌n-Zade, A. N. Varchenko, Singularities of Differentiable Maps I, Monogr. Math. 82, Birkhäuser, Boston, 1985.

[3] J. Basto-Gonçalves, Implicit Hamiltonian equations, Mat. Contemp. 12 (1997), 123-137.

[4] J. W. Bruce, A note on first order differential equations of degree greater than one and wavefront evolution, Bull. London Math. Soc. 16 (1984), 139-144.

[5] I. Ekeland, Discontinuités de champs hamiltoniens et existence de solutions optimales en calcul de variations, Inst. Hautes Études Sci. Publ. Math. 47 (1977), 5-32.

[6] C. G. Gibson, K. Wirthmüller, A. A. du Plessis, E. J. N. Looijenga, Topological Stability of Smooth Mappings, Lecture Notes in Math. 552, Springer, Berlin, 1976.

[7] S. Janeczko, On implicit Lagrangian differential systems, Ann. Polon. Math. 74 (2000), 133-141.

[8] J. Kijowski, W. M. Tulczyjew, A Symplectic Framework for Field Theories, Lecture Notes in Phys. 107, Springer, Berlin, 1979.

[9] I. Kupka, Abnormal extremals, Preprint, Paris VI, 1992.

[10] W. Liu, H. J. Sussmann, Shortest paths for sub-Riemannian metrics on rank-two distributions, Mem. Amer. Math. Soc. 118 (1995), No. 564.

[11] J. Martinet, Singularities of Smooth Functions and Maps, London Math. Soc. Lecture Note Ser. 58, Cambridge Univ. Press, Cambridge, 1982.

[12] R. Montgomery, A survey of singular curves in sub-Riemannian geometry, J. Dynam. Control Systems 1 (1995), 49-90.

[13] F. Pelletier, L. Valère Bouche, Le problème des géodésiques en géométrie sous-riemannienne singulière, C. R. Acad. Sci. Paris Sér. I Math. 317 (1993), 71-76.

[14] F. Pelletier, L. Valère Bouche, Abnormality of trajectory in sub-Riemannian structure, in: Geometry in Nonlinear Control and Differential Inclusions, Banach Center Publ. 32, 1995, 301-317.

[15] S. Pnevmatikos, Structures symplectiques singulières génériques, Ann. Inst. Fourier (Grenoble) 33 (1984), 201-218.

[16] S. Pnevmatikos, D. Pliakis, Quelques propriétés génériques des formes quadratiques, C. R. Acad. Sci. Paris Sér. I Math. 323 (1996), 647-652.

[17] L. S. Pontryagin, V. G. Boltyanskii, R. V. Gamkrelidze, E. F. Mishchenko, The Mathematical Theory of Optimal Processes, Pergamon Press, New York, 1964.

[18] F. Takens, Implicit differential equations: some open problems, in: Singularités d'applications differentiables (Plans-sur-Bex, 1975), Lecture Notes in Math. 535, Springer, Berlin, 1976, 237-253.

[19] R. Thom, Sur les équations différentielles multiformes et leurs intégrales singulières, Colloque E. Cartan, Paris, 1971.

[20] A. Weinstein, Lectures on Symplectic Manifolds, CBMS Regional Conf. Ser. in Math. 29, Amer. Math. Soc., Providence, 1977. 